
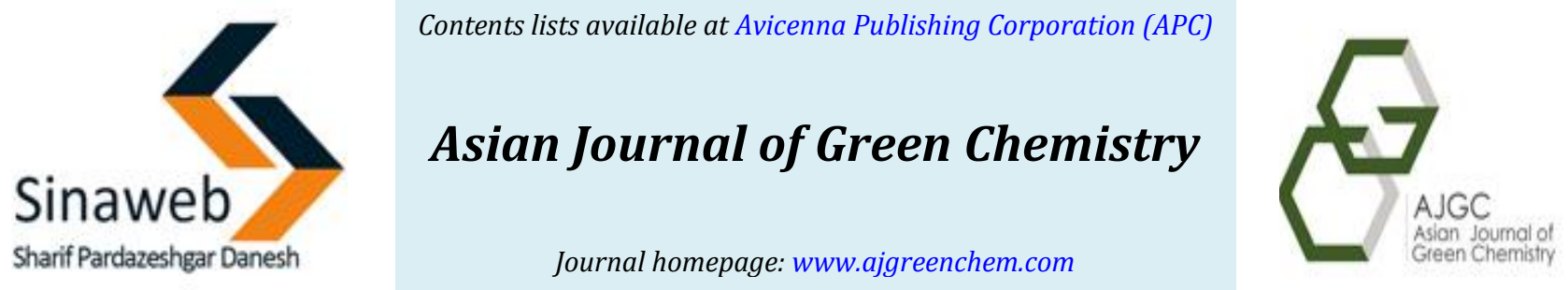

Orginal Research Article

\title{
Quinolinium bound chromium(VI) reagents for efficient electrophilic aromatic nitration and thiocyanation reactions using sodium nitrate and ammonium thiocyanate
} \author{
Yerraguntlab, Ivon Chakravarthi Elisha ${ }^{a}$ \\ a Department of Chemistry, Rayalaseema University, Kurnool-518 007 (A.P.) India \\ b Department of Chemistry, Govt. City College, Hyderabad-500002 (T.S.) India \\ c Department of Chemistry, Osmania University, Hyderabad-500007 (T.S.) India
}

Satish Babu Kodalia,b, Narendar Reddy Jakkuª, , Chinna Rajanna Kamatalac,*, Rajeshwar Rao

\begin{tabular}{|c|c|}
\hline ARTICLE INFORI & ABSTRACT \\
\hline $\begin{array}{l}\text { Received: } 19 \text { October } 2017 \\
\text { Received in revised: } 16 \text { Novamber } 2017 \\
\text { Accepted: } 17 \text { Novamber } 2017 \\
\text { Available online: } 11 \text { December } 2017 \\
\text { DOI: } 10.22631 \text { /ajgc.2017.101557.1031 } \\
\text { KEYWORDS } \\
\text { Quinolinium chlorochromate } \\
\text { Quinolinium dichromate } \\
\text { NaNO }_{2} / \mathrm{KHSO}_{4} \\
\mathrm{Nitration} \\
\mathrm{NH}_{4} \mathrm{SCN} / \mathrm{KHSO}_{4}\end{array}$ & $\begin{array}{l}\text { Quinolinium dichromate (QDC) and quinolinium chlorochromate (QCC) were } \\
\text { studied as efficient reagents for electrophilic aromatic nitration using sodium } \\
\text { nitrite and thiocyanation by using ammonium thiocyanate under } \\
\text { conventional, ultrasonic and solvent-free microwave assisted conditions. The } \\
\text { microwave-assisted protocols exhibited remarkable rate acceleration and } \\
\text { offered selective thiocyanation of the aromatic and heteroaromatic } \\
\text { compounds with good yields. Addition of anionic (SDS) micelle to the } \\
\text { reaction medium afforded considerable rate enhancements, while cationic } \\
\text { (CTAB) and nonionic (Tx-100) micelles did not affect the rate noticeably. } \\
\text { These protocols have were extended to regioselective thiocyanation of } \\
\text { various aromatic compounds. Results were are completely similar to the } \\
\text { nitration protocols. However, the reactions revealed comparatively shorter } \\
\text { reaction times, and better yields in nitration reactions than in thiocyanation } \\
\text { protocols. Catalytic effect of the SDS was attributed to the electrostatic } \\
\text { stabilization of the cationic electrophile }\left(\mathrm{NO}_{2}{ }^{+} \text {in nitration, and SCN }{ }^{+} \text {in }\right. \\
\text { thiocyanation). }\end{array}$ \\
\hline
\end{tabular}

Corresponding author, email: kcrajannaou@yahoo.com (K.C. Rajanna).

Tel.: +989190305453. 


\section{Graphical Abstract}

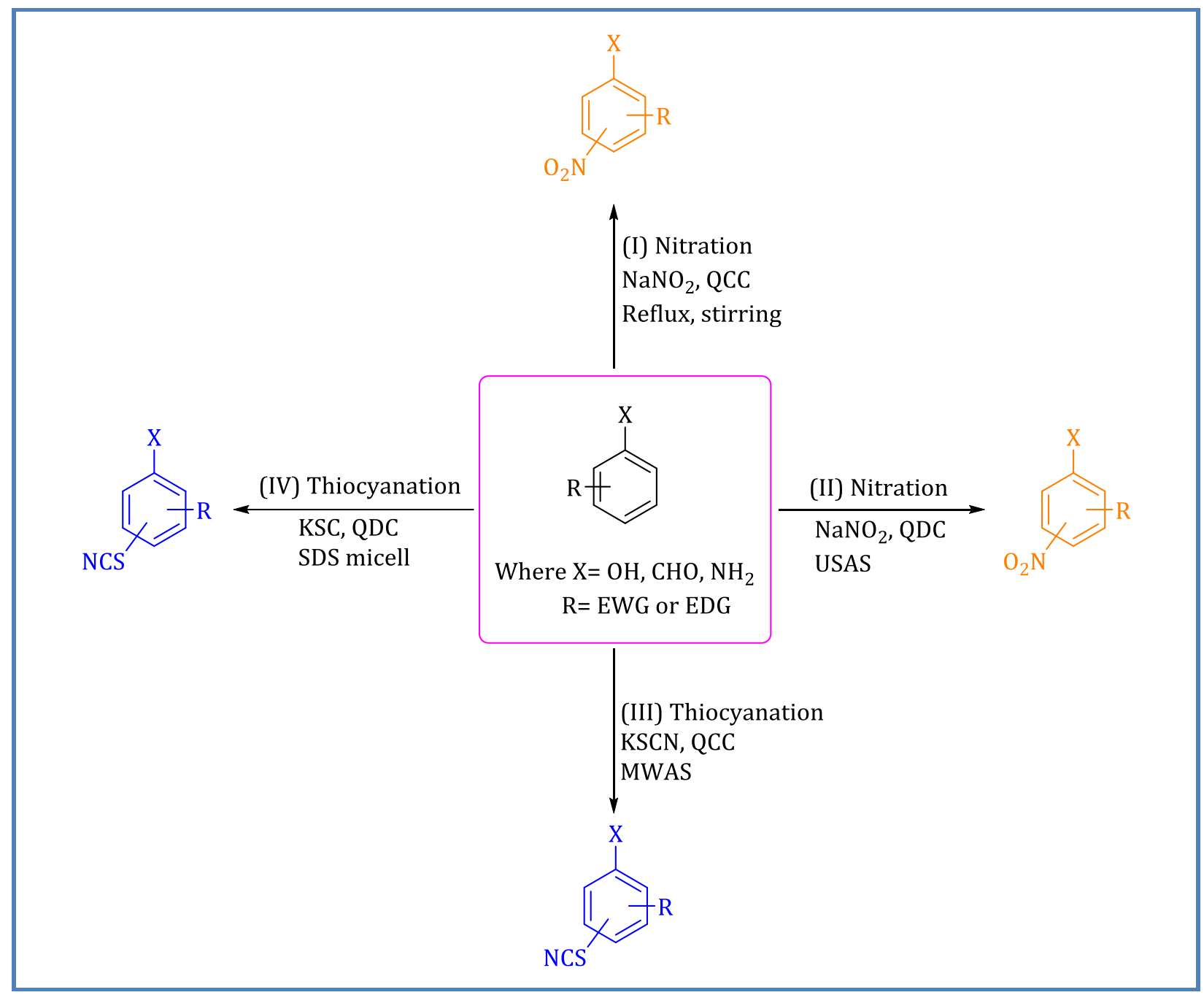

\section{Introduction}

Electrophilic aromatic substitution (EAS) reactions occupy a prime place in synthetic organic chemistry, which introduce different functional groups on an aromatic ring system [1, 2]. Overall electrophilic aromatic substitution reaction involves the substitution of hydrogen (or sometimes multiple $\mathrm{H}$ ) on the aromatic ring system by an electrophile, designated $\mathrm{E}^{+}$.

Nitration and thiocyanation of the aromatic and heteroaromatic compounds are typical electrophilic substitution reactions which affect the formation of carbon-heteroatom ( $\mathrm{C}-\mathrm{N}$, and $\mathrm{C}-\mathrm{S}$ ) bond formation reactions in the organic synthesis. Compounds obtained by these reactions are useful as precursors for the production of pharmaceutical, agrochemical and industrial products. Over the years, several commercial processes were developed to produce such materials. However, many of the classical protocols pertaining to the nitration and thiocyanation procedures commonly lead to 
mixtures of regioisomers. The unspent acids left out in these protocols often cause environmental pollution. In recent years, several new approaches have been developed to control the regiochemistry of the reactions [3-20].

Recently, we have accomplished the use of quinolinium dichromate and quinolinium chlorochromate as efficient catalysts to trigger oxidative bromination and iodination of aromatic hydrocarbons with $\mathrm{KBr} / \mathrm{KI}$ and $\mathrm{KHSO}_{4}$ under acid-free conditions. Reaction times reduced significantly under the sonication, followed by corresponding mono bromo derivatives with a good yield and high regioselectivity [21]. In the present study, the authors embarked on exploring quinolinium dichromate and quinolinium chlorochromate as efficient reagents to trigger in situ electrophilic nitration and thiocyanation of aromatic compounds using sodium nitrite and thiocyanate respectively. Neverthless, sevral "Onium halochromates and dichromates" were earlier explored for oxidation as well as oxyhalogenation of the organic compounds [22-24].

In this study, the authors also explored the use of micelle forming surfactants, ultrasound and microwave to assist the QCC and QDC mediated reactions with a view to accelerate reaction rates for achieving better yields as well as improving the greenery of the reaction protocols (Scheme 1) [25].

\section{Experimental}

\section{Matreials and methods}

Acetonitrile (MeCN) was purified according to the standard literature procedures [26]. Binary compositions of the acetonitrile and water were used as solvent in the present investigation. Laboratory distilled water was further purified over alkaline $\mathrm{KMnO}_{4}$ and acidified $\mathrm{K}_{2} \mathrm{Cr}_{2} \mathrm{O}_{7}$ in a sequence. Quinolinium dichromate $\left(\left[\left(\mathrm{C}_{9} \mathrm{H}_{7} \mathrm{NH}^{+}\right)_{2} \mathrm{Cr}_{2} \mathrm{O}_{7}\right]\right.$ or QDC) was prepared according to the method of Bala Subramanian et al. [22, 23]. Quinolinium chlorochromate (QCC) was prepared according to the reported method of Singh et al. [24]. The purity of the QCC was checked by iodometric method, according to standard procedures. The other chemicals used were of either Analar BDH or Merck samples.

General procedure for aromatic nitration and thiocyanation under conventional conditions using (QDC/QCC) reagent

A centimolar (0.01 mol) organic substrate, $0.02 \mathrm{~mol}$ of $\mathrm{NaNO}_{2}$ and about 0.015 moles of $\mathrm{Cr}(\mathrm{VI})$ reagent (QDC/QCC), about $50 \mathrm{mg}$ of $\mathrm{KHSO}_{4}$, and solvent (MeCN) were taken in a previously cleaned in a round bottom flask and stirred for about 1 to $2 \mathrm{~h}$ at room temperature. After completion of the reaction, as confirmed by TLC, the reaction mixture is treated with $5 \%$ sodium thiosulfate solution, 
Scheme 1. Structure of QDC and QCC

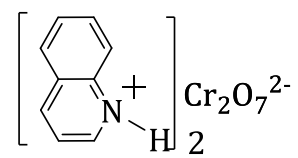

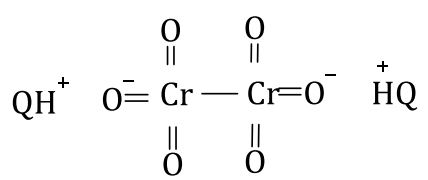

Quinolinium dichromate (QDC)

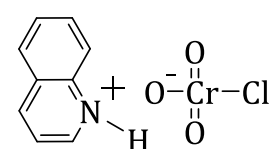

$\mathrm{QH}^{+} \stackrel{\mathrm{O}}{\mathrm{O}-\stackrel{\mathrm{O}}{\mathrm{C}} \mathrm{r}-\mathrm{Cl}}$

Quinoliniumchlorochromate (QCC)

followed by the addition of ethyl acetate. The organic layer was separated, dried over $\mathrm{Na}_{2} \mathrm{SO}_{4}$ and evaporated under vacuum. Then, it was purified by column chromatography using pet-ether and ethyl acetate to get pure product. In case of nitration of aromatic compounds, nitroaromatic derivatives were obtained, and characterized by the spectroscopic analysis. Procedure for thiocyanation reactions is almost similar, and the reactions are carried out with $0.02 \mathrm{~mol}_{\text {of }} \mathrm{NH}_{4} \mathrm{SCN}$.

\section{General procedure for aromatic nitration and thiocyanation under sonication}

Methodology for the ultrasonically assisted reactions are similar to the conventional methods. Organic substrate, $\mathrm{NaNO}_{2}$, oniumCr(VI) reagent (QDC/QCC), about $50 \mathrm{mg}$ of $\mathrm{KHSO}_{4}$, and solvent (MeCN) were taken in a clean conical flask at room temperature and immersed in a sonicator. Progress of the reaction was checked by TLC. After completion, the reaction mixture is further processed for the isolation of product as detailed in earlier sections. For thiocyanation reactions 0.02 mol of $\mathrm{NH}_{4} \mathrm{SCN}$ is used under otherwise similar conditions.

General procedure for microwave assisted aromatic nitration and thiocyanation under solvent-free MW conditions

Organic substrate $(0.01 \mathrm{~mol}), \mathrm{NaNO}_{2}(0.02 \mathrm{~mol})$, about $0.015 \mathrm{~mol}$ of $\mathrm{Cr}(\mathrm{VI})$ reagent (QDC/QCC), about $50 \mathrm{mg}$ of $\mathrm{KHSO}_{4}$, and silicagel were mixed thoroughly in a beaker. The resulting reaction mixture was placed in a controlled microwave synthesizer (Biotage Initiator + SP Wave model- 0.200 $\mathrm{W}$ at $2.45 \mathrm{GHz}$, capped at $60 \mathrm{~W}$ during steady state) for a few minutes (attains temperature $100{ }^{\circ} \mathrm{C}$ and 2 bar pressure) till the reaction is completed. Progress of the reaction was monitored by TLC. After completion, the reaction mixture is further processed for the isolation of product as detailed in earlier sections. For thiocyanation reactions $0.02 \mathrm{~mol}$ of $\mathrm{NH}_{4} \mathrm{SCN}$ is used under otherwise similar conditions.

General procedure for aromatic nitration and thiocyanation under micellar conditions using $(Q D C / Q C C)$ reagent

A centimolar $(0.01 \mathrm{~mol})$ organic substrates $0.01 \mathrm{~mol}$ of $\mathrm{NaNO}_{2}$ and about 0.015 moles of Onium 
Cr(VI) reagent (QDC/QCC), micelle forming surfactant (5×5 ML) of $0.05 \mathrm{M}$ cetyltrimethyl ammonium bromide (CTAB), sodium dodecylsulfate (SDS) or Triton-X-100), about $50 \mathrm{mg}$ of $\mathrm{KHSO}_{4}$, and solvent $(\mathrm{MeCN})$ were taken in a previously cleaned round bottom flask and stirred for about 1 to 2 hours at room temperature. After completion of the reaction, as confirmed by TLC, the reaction mixture was treated with $5 \%$ sodium thiosulfate solution, followed by the addition of ethyl acetate. The organic layer was separated, dried over $\mathrm{Na}_{2} \mathrm{SO}_{4}$ and evaporated under vacuum, purified by column chromatography using pet-ether and ethyl acetate to get the pure product. In case of nitration of aromatic compounds, nitroaromatic derivatives were produced and analyzed from spectroscopic studies. However, for thiocyanation reactions $0.02 \mathrm{~mol}$ of $\mathrm{NH}_{4} \mathrm{SCN}$ are used under otherwise similar conditions.

\section{Results and discussion}

Earlier reviews and publications on micelle mediated [27-31], ultrasonic [32, 33] and microwave [34-36] assisted organic synthesis revealed that these protocols satisfy both economic and environmental demands, as recommended by Paul Anestas and John Walter in the green chemistry formulations [25].

Quinolinium chlorochromate/ $\mathrm{NaNO}_{2}$ and quinolinium dichromate/NaNO 2 triggered nitration of aromatic compounds

The nitration reactions of aromatic compounds were conducted using (QDC)/ $\mathrm{NaNO}_{2}$, and $(\mathrm{QCC}) / \mathrm{NaNO}_{2}$ combinations in the aqueous $\mathrm{KHSO}_{4}$ under the mineral acid free conditions (Scheme 2). The described methods worked out well for an array of functionalities including phenols, and anilines. The yields of the major products are compiled in Table 1,2, and 3. The nitration of aromatic compounds required 4-5 $\mathrm{h}$ under the conventional conditions at reflux temperatures. However, under the sonication, the reaction times were drastically reduced to about 30-40 min followed by considerable yield enhancements.

Quinolinium chlorochromate/ $\mathrm{NH}_{4} \mathrm{SCN}$ and quinolinium dichromate/ $\mathrm{NH}_{4} \mathrm{SCN}$ triggred thiocyanation of aromatic compounds

Quinolinium dichromate (QDC)/ $\mathrm{NH}_{4} \mathrm{SCN}$, and quinolinium chlorochromate (QCC)/ $\mathrm{NH}_{4} \mathrm{SCN}$ reagents rtriggered thiocyanation of the aromatic compounds in aqueous $\mathrm{KHSO}_{4}$ under mineral acid-free conditions. Aromatic compounds such as phenols, anilines and certain heteroaromatic compounds were used for thiocyanation as shown in Scheme 2. The thiocyanation reactions were generally more sluggish than the nitration reactions, which required more than $10 \mathrm{~h}$ under the conventional conditions at reflux temperatures (Table 4 and 5). But, under the sonication, the reaction times were considerably reduced to about 25-40 min followed by a significant yield enhancements. Comparing the reaction times and product yields 
presented in Table 4 and 5 revealed that QDC mediated reactions are generally faster than the corresponding QCC mediated reactions.

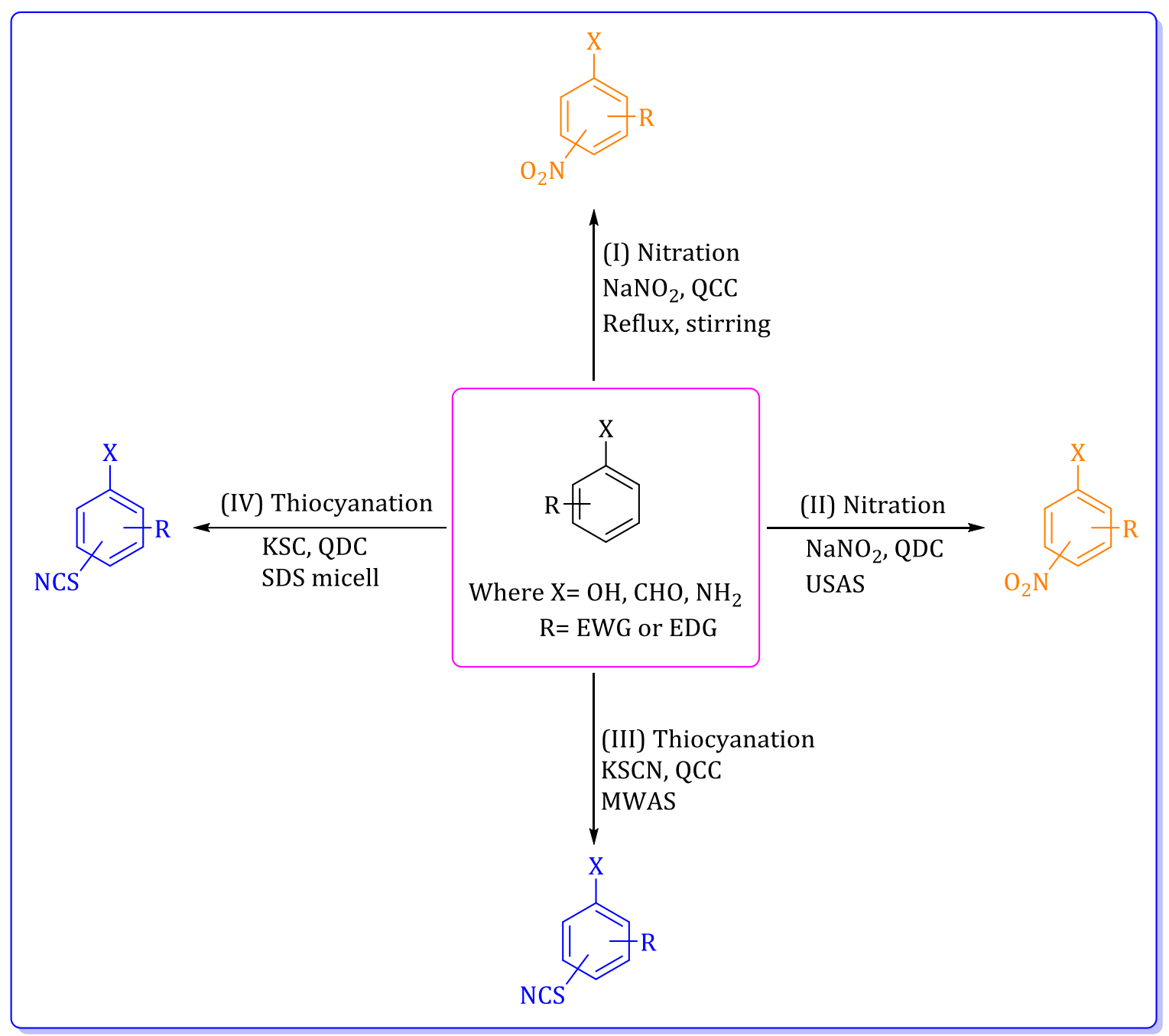

Scheme 2. QCC/QDC mediated electrophilic aromatic substitution reactions under different conditions

Table 1. Nitration of aromatic compounds in presence of $\mathrm{QCC} / \mathrm{NaNO}_{2}$

\begin{tabular}{|c|c|c|c|c|c|c|c|}
\hline \multirow[b]{2}{*}{ Entry } & \multirow[b]{2}{*}{ Product } & \multicolumn{2}{|c|}{ Conventional } & \multicolumn{2}{|c|}{ USAR } & \multicolumn{2}{|c|}{ MWA R } \\
\hline & & $\begin{array}{l}\text { Time } \\
(\min )\end{array}$ & $\begin{array}{c}\text { Yield } \\
(\%)\end{array}$ & $\begin{array}{l}\text { Time } \\
\text { (min) }\end{array}$ & $\begin{array}{l}\text { Yield } \\
(\%)\end{array}$ & $\begin{array}{l}\text { Time } \\
\text { (sec) }\end{array}$ & $\begin{array}{c}\text { Yield } \\
(\%)\end{array}$ \\
\hline 1 & $2-\mathrm{NO}_{2} \mathrm{C}_{6} \mathrm{H}_{4} \mathrm{OH}$ & 60 & 70 & 25 & 73 & 150 & 75 \\
\hline 2 & $2-\mathrm{CH}_{3}-4-\mathrm{NO}_{2} \mathrm{C}_{6} \mathrm{H}_{3} \mathrm{OH}$ & 60 & 68 & 25 & 69 & 150 & 75 \\
\hline 3 & $4-\mathrm{CH}_{3}-2-\mathrm{NO}_{2} \mathrm{C}_{6} \mathrm{H}_{3} \mathrm{OH}$ & 60 & 65 & 25 & 72 & 150 & 73 \\
\hline 4 & $3-\mathrm{CH}_{3}-4-\mathrm{NO}_{2} \mathrm{C}_{6} \mathrm{H}_{3} \mathrm{OH}$ & 60 & 70 & 25 & 70 & 150 & 72 \\
\hline 5 & $4-\mathrm{NO}_{2} 2-\mathrm{Cl} \mathrm{C}_{6} \mathrm{H}_{3} \mathrm{OH}$ & 65 & 62 & 30 & 70 & 180 & 68 \\
\hline 6 & $2-\mathrm{NO}_{2} 4-\mathrm{Cl} \mathrm{C} \mathrm{C}_{6} \mathrm{H}_{3} \mathrm{OH}$ & 70 & 60 & 30 & 65 & 120 & 68 \\
\hline 7 & $2-\mathrm{NO}_{2} 4-\mathrm{Br} \mathrm{C}{ }_{6} \mathrm{H}_{3} \mathrm{OH}$ & 60 & 65 & 35 & 68 & 120 & 70 \\
\hline
\end{tabular}




\begin{tabular}{|c|c|c|c|c|c|c|c|}
\hline 8 & 2- $\mathrm{NO}_{2} \mathrm{C}_{6} \mathrm{H}_{3}-1,4-\mathrm{OH}$ & 50 & 72 & 20 & 70 & 120 & 70 \\
\hline 9 & 2- $\mathrm{NO}_{2}-1-\mathrm{Naph}$ thol & 55 & 70 & 25 & 72 & 120 & 75 \\
\hline 10 & 1-NO $\mathrm{N}_{2}-2-\mathrm{Naphthol}$ & 60 & 70 & 25 & 68 & 120 & 72 \\
\hline 11 & $3-\mathrm{NO}_{2} \mathrm{C}_{6} \mathrm{H}_{4} \mathrm{NH}_{2}$ & 185 & 74 & 40 & 77 & 180 & 82 \\
\hline 12 & $3-\mathrm{NO}_{2} \mathrm{C}_{6} \mathrm{H}_{4} \mathrm{NH}_{2}$ & 190 & 73 & 60 & 79 & 180 & 82 \\
\hline 13 & $\begin{array}{l}\text { 3-OH-4- } \mathrm{NO}_{2} \\
\text { acetophenone }\end{array}$ & 65 & 60 & 40 & 65 & 120 & 65 \\
\hline 14 & $2,-4-\mathrm{NO}_{2} \mathrm{C}_{6} \mathrm{H}_{4} \mathrm{OH}$ & 190 & 73 & 50 & 75 & 180 & 80 \\
\hline 15 & 4-Cl-3- $\mathrm{NO}_{2} \mathrm{C}_{6} \mathrm{H}_{3} \mathrm{CHO}$ & 80 & 60 & 45 & 62 & 120 & 65 \\
\hline 16 & $2-\mathrm{OH}-5-\mathrm{NO}_{2}-\mathrm{C}_{6} \mathrm{H}_{3} \mathrm{CHO}$ & 80 & 65 & 45 & 62 & 120 & 60 \\
\hline 17 & $4-\mathrm{OH}-3-\mathrm{NO}_{2}-\mathrm{C}_{6} \mathrm{H}_{3} \mathrm{CHO}$ & 80 & 60 & 40 & 65 & 120 & 65 \\
\hline 18 & 3-NO2- $\mathrm{C}_{6} \mathrm{H}_{4} \mathrm{COOH}$ & 190 & 76 & 60 & 75 & 180 & 75 \\
\hline 19 & $2-\mathrm{NO}_{2}$ furan & 170 & 69 & 50 & 73 & 180 & 77 \\
\hline 20 & 2- $\mathrm{NO}_{2}$ thiophene & 190 & 73 & 60 & 75 & 180 & 79 \\
\hline 21 & 1- $\mathrm{NO}_{2}$ Naphthalene & 200 & 74 & 55 & 76 & 220 & 80 \\
\hline 22 & 1-Cl, 2- $\mathrm{NO}_{2} \mathrm{C}_{6} \mathrm{H}_{4}$ & 180 & 76 & 40 & 75 & 180 & 80 \\
\hline 23 & $1-\mathrm{Br}, 2-\mathrm{NO}_{2} \mathrm{C}_{6} \mathrm{H}_{4}$ & 190 & 75 & 50 & 76 & 220 & 78 \\
\hline 24 & 1- $\mathrm{CH}_{3}, 2-\mathrm{NO}_{2} \mathrm{C}_{6} \mathrm{H}_{4}$ & 180 & 75 & 55 & 74 & 180 & 78 \\
\hline 25 & 2- $\mathrm{NO}_{2} \mathrm{C}_{6} \mathrm{H}_{4} \mathrm{CH}_{2} \mathrm{OH}$ & 190 & 76 & 60 & 77 & 230 & 78 \\
\hline 26 & $2-\mathrm{OH} 4-\mathrm{NO}_{2} \mathrm{C}_{6} \mathrm{H}_{3} \mathrm{COOH}$ & 200 & 74 & 60 & 78 & 230 & 78 \\
\hline 27 & $3-\mathrm{NO}_{2} \mathrm{C}_{6} \mathrm{H}_{4} \mathrm{CN}$ & 220 & 76 & 80 & 76 & 300 & 80 \\
\hline 28 & $3-\mathrm{NO}_{2} \quad \mathrm{C}_{6} \mathrm{H}_{5} \mathrm{CONH}_{2}$ & 240 & 72 & 84 & 76 & 300 & 78 \\
\hline
\end{tabular}

Table 2. Nitration of aromatic compounds in presence of $\mathrm{QDC} / \mathrm{NaNO}_{2}$

\begin{tabular}{|c|c|c|c|c|c|c|c|}
\hline \multirow[b]{2}{*}{ Entry } & \multirow[b]{2}{*}{ Product } & \multicolumn{2}{|c|}{ Conventional } & \multicolumn{2}{|c|}{ USAR } & \multicolumn{2}{|c|}{ MWA R } \\
\hline & & $\begin{array}{l}\text { Time } \\
\text { (min) }\end{array}$ & $\begin{array}{c}\text { Yield } \\
(\%)\end{array}$ & $\begin{array}{l}\text { Time } \\
\text { (min) }\end{array}$ & $\begin{array}{c}\text { Yield } \\
(\%)\end{array}$ & $\begin{array}{l}\text { Time } \\
\text { (sec) }\end{array}$ & $\begin{array}{c}\text { Yield } \\
(\%)\end{array}$ \\
\hline 1 & $2-\mathrm{NO}_{2} \mathrm{C}_{6} \mathrm{H}_{4} \mathrm{OH}$ & 45 & 70 & 20 & 73 & 130 & 75 \\
\hline 2 & $2-\mathrm{CH}_{3}-4-\mathrm{NO}_{2} \quad \mathrm{C}_{6} \mathrm{H}_{3} \mathrm{OH}$ & 45 & 68 & 20 & 69 & 130 & 75 \\
\hline 3 & $4-\mathrm{CH}_{3}-2-\mathrm{NO}_{2} \quad \mathrm{C}_{6} \mathrm{H}_{3} \mathrm{OH}$ & 45 & 65 & 20 & 72 & 135 & 73 \\
\hline 4 & $3-\mathrm{CH}_{3}-4-\mathrm{NO}_{2} \quad \mathrm{C}_{6} \mathrm{H}_{3} \mathrm{OH}$ & 45 & 70 & 23 & 70 & 140 & 72 \\
\hline 5 & $4-\mathrm{NO}_{2}-2-\mathrm{Cl} \mathrm{C}_{6} \mathrm{H}_{3} \mathrm{OH}$ & 50 & 62 & 26 & 70 & 160 & 68 \\
\hline 6 & $2-\mathrm{NO}_{2} 4-\mathrm{Cl} \mathrm{C}{ }_{6} \mathrm{H}_{3} \mathrm{OH}$ & 50 & 60 & 25 & 65 & 110 & 68 \\
\hline 7 & $2-\mathrm{NO}_{2}-4-\mathrm{Br} \quad \mathrm{C}_{6} \mathrm{H}_{3} \mathrm{OH}$ & 55 & 65 & 30 & 68 & 105 & 70 \\
\hline 8 & $2-\mathrm{NO}_{2}-\mathrm{C}_{6} \mathrm{H}_{3}-1,4-\mathrm{OH}$ & 40 & 72 & 20 & 70 & 110 & 70 \\
\hline 9 & 2- $\mathrm{NO}_{2}$-1-Naphthol & 45 & 70 & 23 & 72 & 110 & 75 \\
\hline 10 & 1-NO $2-2-\mathrm{Naphthol}$ & 45 & 70 & 22 & 68 & 110 & 72 \\
\hline 11 & $3-\mathrm{NO}_{2} \mathrm{C}_{6} \mathrm{H}_{4} \mathrm{NH}_{2}$ & 60 & 74 & 35 & 77 & 160 & 82 \\
\hline 12 & $3-\mathrm{NO}_{2} \mathrm{C}_{6} \mathrm{H}_{4} \mathrm{NH}_{2}$ & 80 & 73 & 55 & 79 & 165 & 82 \\
\hline 13 & $\begin{array}{l}\text { 3-OH-4- } \mathrm{NO}_{2}- \\
\text { acetophenone }\end{array}$ & 60 & 60 & 35 & 65 & 115 & 65 \\
\hline 14 & $2,-4-\mathrm{NO}_{2} \mathrm{C}_{6} \mathrm{H}_{4} \mathrm{OH}$ & 70 & 73 & 45 & 75 & 165 & 80 \\
\hline 15 & $4-\mathrm{Cl}-3-\mathrm{NO}_{2} \mathrm{C}_{6} \mathrm{H}_{3} \mathrm{CHO}$ & 65 & 60 & 40 & 62 & 110 & 65 \\
\hline 16 & $\begin{array}{c}2-\mathrm{OH}-5-\mathrm{NO}_{2-} \\
\mathrm{C}_{6} \mathrm{H}_{3} \mathrm{CHO}\end{array}$ & 65 & 65 & 40 & 62 & 114 & 60 \\
\hline 17 & $\begin{array}{c}\text { 4-OH-3-NO }-\mathrm{N}_{2}- \\
\mathrm{C}_{6} \mathrm{H}_{3} \mathrm{CHO}\end{array}$ & 60 & 60 & 35 & 65 & 115 & 65 \\
\hline
\end{tabular}




\begin{tabular}{|c|c|c|c|c|c|c|c|}
\hline 18 & $3-\mathrm{NO} 2-\mathrm{C}_{6} \mathrm{H}_{4} \mathrm{COOH}$ & 160 & 76 & 50 & 75 & 160 & 75 \\
\hline 19 & $2-\mathrm{NO}_{2}$ furan & 140 & 69 & 45 & 73 & 155 & 77 \\
\hline 20 & $2-\mathrm{NO}_{2}$ thiophene & 150 & 73 & 50 & 75 & 160 & 79 \\
\hline 21 & 1- $\mathrm{NO}_{2}$ Naphthalene & 175 & 74 & 50 & 76 & 200 & 80 \\
\hline 22 & $1-\mathrm{Cl}, 2-\mathrm{NO}_{2} \mathrm{C}_{6} \mathrm{H}_{4}$ & 160 & 76 & 35 & 75 & 160 & 80 \\
\hline 23 & $1-\mathrm{Br}, 2-\mathrm{NO}_{2} \mathrm{C}_{6} \mathrm{H}_{4}$ & 150 & 75 & 40 & 76 & 213 & 78 \\
\hline 24 & $1-\mathrm{CH}_{3}, 2-\mathrm{NO}_{2} \mathrm{C}_{6} \mathrm{H}_{4}$ & 140 & 75 & 45 & 74 & 158 & 78 \\
\hline 25 & 2- $\mathrm{NO}_{2} \mathrm{C}_{6} \mathrm{H}_{4} \mathrm{CH}_{2} \mathrm{OH}$ & 130 & 76 & 50 & 77 & 200 & 78 \\
\hline 26 & $\begin{array}{l}2-\mathrm{OH} 4-\mathrm{NO}_{2} \\
\mathrm{C}_{6} \mathrm{H}_{3} \mathrm{COOH}\end{array}$ & 180 & 74 & 50 & 78 & 200 & 78 \\
\hline 27 & $3-\mathrm{NO}_{2} \mathrm{C}_{6} \mathrm{H}_{4} \mathrm{CN}$ & 185 & 76 & 65 & 76 & 260 & 80 \\
\hline 28 & $3-\mathrm{NO}_{2} \quad \mathrm{C}_{6} \mathrm{H}_{5} \mathrm{CONH}_{2}$ & 190 & 72 & 74 & 76 & 260 & 78 \\
\hline
\end{tabular}

Table 3. $\mathrm{QDC} / \mathrm{NaNO}_{2}$ and $\mathrm{QCC} / \mathrm{NaNO}_{2}$ mediated nitration of aromatic compounds in SDS medium

\begin{tabular}{|c|c|c|c|c|c|}
\hline \multirow[t]{2}{*}{ Entry } & \multirow[t]{2}{*}{ Product } & \multicolumn{2}{|c|}{$\mathrm{QCC} / \mathrm{NaNO}_{2}$} & \multicolumn{2}{|c|}{$\mathrm{QDC} / \mathrm{NaNO}_{2}$} \\
\hline & & Time (min) & Yield (\%) & $\mathrm{RT}(\min )$ & Yield (\%) \\
\hline 1 & $2-\mathrm{NO}_{2} \mathrm{C}_{6} \mathrm{H}_{4} \mathrm{OH}$ & 45 & 73 & 35 & 83 \\
\hline 2 & $2-\mathrm{CH}_{3}-4-\mathrm{NO}_{2} \mathrm{C}_{6} \mathrm{H}_{3} \mathrm{OH}$ & 45 & 69 & 33 & 79 \\
\hline 3 & $4-\mathrm{CH}_{3}-2-\mathrm{NO}_{2} \mathrm{C}_{6} \mathrm{H}_{3} \mathrm{OH}$ & 45 & 72 & 35 & 80 \\
\hline 4 & $3-\mathrm{CH}_{3}-4-\mathrm{NO}_{2} \mathrm{C}_{6} \mathrm{H}_{3} \mathrm{OH}$ & 45 & 70 & 35 & 75 \\
\hline 5 & $4-\mathrm{NO}_{2} 2-\mathrm{Cl} \mathrm{C}_{6} \mathrm{H}_{3} \mathrm{OH}$ & 50 & 70 & 40 & 75 \\
\hline 6 & $2-\mathrm{NO}_{2} 4-\mathrm{Cl} \mathrm{C} \mathrm{C}_{6} \mathrm{H}_{3} \mathrm{OH}$ & 50 & 65 & 40 & 70 \\
\hline 7 & $2-\mathrm{NO}_{2} 4-\mathrm{Br} \mathrm{C}_{6} \mathrm{H}_{3} \mathrm{OH}$ & 55 & 68 & 45 & 78 \\
\hline 8 & 2- $\mathrm{NO}_{2} \mathrm{C}_{6} \mathrm{H}_{3}-1,4-\mathrm{OH}$ & 40 & 70 & 30 & 76 \\
\hline 9 & 2- $\mathrm{NO}_{2}$-1-Naphthol & 45 & 72 & 35 & 79 \\
\hline 10 & 1-NO -2 -Naphthol & 45 & 68 & 35 & 73 \\
\hline 11 & $3-\mathrm{NO}_{2} \mathrm{C}_{6} \mathrm{H}_{4} \mathrm{NH}_{2}$ & 60 & 77 & 55 & 82 \\
\hline 12 & $3-\mathrm{NO}_{2} \mathrm{C}_{6} \mathrm{H}_{4} \mathrm{NH}_{2}$ & 80 & 79 & 70 & 85 \\
\hline 13 & $\begin{array}{l}\text { 3-OH-4- }-\mathrm{NO}_{2}- \\
\text { acetophenone }\end{array}$ & 60 & 65 & 50 & 75 \\
\hline 14 & $2,-4-\mathrm{NO}_{2} \mathrm{C}_{6} \mathrm{H}_{4} \mathrm{OH}$ & 70 & 75 & 60 & 78 \\
\hline 15 & $4-\mathrm{Cl}-3-\mathrm{NO}_{2} \mathrm{C}_{6} \mathrm{H}_{3} \mathrm{CHO}$ & 65 & 62 & 55 & 72 \\
\hline 16 & $2-\mathrm{OH}-5-\mathrm{NO}_{2}-\mathrm{C}_{6} \mathrm{H}_{3} \mathrm{CHO}$ & 65 & 62 & 55 & 76 \\
\hline 17 & $4-\mathrm{OH}-3-\mathrm{NO}_{2}-\mathrm{C}_{6} \mathrm{H}_{3} \mathrm{CHO}$ & 60 & 65 & 50 & 74 \\
\hline 18 & $3-\mathrm{NO} 2-\mathrm{C}_{6} \mathrm{H}_{4} \mathrm{COOH}$ & 80 & 75 & 65 & 81 \\
\hline 19 & 2- $\mathrm{NO}_{2}$ furan & 70 & 73 & 58 & 78 \\
\hline 20 & 2- $\mathrm{NO}_{2}$ thiophene & 80 & 75 & 68 & 78 \\
\hline 21 & 1- $\mathrm{NO}_{2}$ Naphthalene & 75 & 76 & 65 & 82 \\
\hline 22 & 1-Cl, $2-\mathrm{NO}_{2} \mathrm{C}_{6} \mathrm{H}_{4}$ & 60 & 75 & 50 & 84 \\
\hline 23 & 1-Br, 2- $\mathrm{NO}_{2} \mathrm{C}_{6} \mathrm{H}_{4}$ & 70 & 76 & 60 & 82 \\
\hline 24 & 1- $\mathrm{CH}_{3}, 2-\mathrm{NO}_{2} \mathrm{C}_{6} \mathrm{H}_{4}$ & 75 & 74 & 55 & 79 \\
\hline 25 & 2- $\mathrm{NO}_{2} \mathrm{C}_{6} \mathrm{H}_{4} \mathrm{CH}_{2} \mathrm{OH}$ & 80 & 77 & 65 & 83 \\
\hline 26 & $2-\mathrm{OH} 4-\mathrm{NO}_{2} \quad \mathrm{C}_{6} \mathrm{H}_{3} \mathrm{COOH}$ & 80 & 78 & 75 & 84 \\
\hline 27 & $3-\mathrm{NO}_{2} \mathrm{C}_{6} \mathrm{H}_{4} \mathrm{CN}$ & 80 & 76 & 65 & 82 \\
\hline 28 & $3-\mathrm{NO}_{2} \quad \mathrm{C}_{6} \mathrm{H}_{5} \mathrm{CONH}_{2}$ & 84 & 76 & 75 & 82 \\
\hline
\end{tabular}


Mechanism of the substitution reactions

It is well established in earlier reports that in aqueous acid media potassium dichromate $\left(\mathrm{K}_{2} \mathrm{Cr}_{2} \mathrm{O}_{7}\right)$ or chromium(VI) exists in several reactive forms such as $\mathrm{HCrO}_{4}^{-}, \mathrm{H}_{2} \mathrm{CrO}_{4},\left[\mathrm{HCrO}_{3}\right]^{+}$, and $\mathrm{HCrO}_{3} \mathrm{~B}$ (where $\mathrm{B}=\mathrm{HSO}_{4}^{-}, \mathrm{ClO}_{4^{-}}$or $\mathrm{NO}_{2}^{-}$). Since the quinolinium dichromate (QDC) is related to $\mathrm{K}_{2} \mathrm{Cr}_{2} \mathrm{O}_{7}$, we have formulated similar types of reactive species with quinolinium ion background [26] according to the following equilibria.

$$
(\mathrm{QH})_{2} \mathrm{Cr}_{2} \mathrm{O}_{7}+\mathrm{H}_{2} \mathrm{O} \rightleftharpoons 2\left[(\mathrm{QH})^{+}\left(\mathrm{HCrO}_{4}\right)^{-}\right]
$$

The QDC may further protonated to from active [(QH)+( $\left.\left.\mathrm{HCrO}_{4}\right)^{-}\right]$(Quinolinium bound chromic acid) species, using the $\left(\mathrm{H}^{+}\right)$thus released from the dissociation of $\mathrm{HSO}_{4}{ }^{-}$, as shown below

$$
\begin{gathered}
\mathrm{HSO}_{4^{-}} \rightleftharpoons \mathrm{SO}_{4}{ }^{2-}+\mathrm{H}^{+} \\
(\mathrm{QH})^{+}\left(\mathrm{HCrO}_{4}\right)^{-}+\mathrm{H}^{+} \rightleftharpoons\left[(\mathrm{QH}) \mathrm{CrO}_{3}\right]^{+}+\mathrm{H}_{2} \mathrm{O}
\end{gathered}
$$

Active species, thus formed may further react with nitrite ion to afford $\left[(\mathrm{QH}) \mathrm{CrO}_{3} \mathrm{NO}_{2}\right]$ species, since the reactions are conducted in excess $\mathrm{NaNO}_{2}$.

$$
\left[(\mathrm{QH}) \mathrm{CrO}_{3}\right]^{+}+\mathrm{NO}_{2^{-}} \rightleftharpoons\left[(\mathrm{QH}) \mathrm{OCrO}_{2} \mathrm{NO}_{2}\right]
$$

Similar type of reactive species could be formed with thiocyanide ( $\mathrm{SCN}^{-}$) ion to afford $\left[(\mathrm{QH}) \mathrm{CrO}_{3}(\mathrm{SCN})\right]$ species, since the reactions are conducted in excess of $\mathrm{NH}_{4} \mathrm{SCN}$. Finally, aromatic substrates undergo electrophilic substitution when the in situ formed electrophile $\left(\mathrm{NO}_{2}{ }^{+}\right.$or $\left.\mathrm{SCN}^{+}\right)$attacks on aromatic ring as shown in the following schematic steps.

$$
\left[(\mathrm{QH}) \mathrm{CrO}_{3}\right]^{++}\left(\mathrm{SCN}^{-}\right) \rightleftharpoons\left[(\mathrm{QH}) \mathrm{CrO}_{3}(\mathrm{SCN})\right]
$$

On the otherhand, the most plausible mechanism in QCC (Quinolinium chlorochromate) triggered reactions could be proposed by considering $(\mathrm{QHOCr}(\mathrm{OH}) \mathrm{Cl})^{+}$(the protonated form of QCC), which is formed according to the following equilibrium:

$$
(\mathrm{QHOCrO} 2 \mathrm{Cl})+\mathrm{H}^{+} \rightleftharpoons(\mathrm{QHOCr}(\mathrm{OH}) \mathrm{OCl})^{+}
$$

The protonated QCC species thus formed $(\mathrm{QHOCr}(\mathrm{OH}) \mathrm{OCl})+$ being a stronger electrophile, may further react with nitrite ion to afford $\left[\mathrm{QHOCr}(\mathrm{OH})(\mathrm{Cl}) \mathrm{NO}_{2}\right]$ species, since the reactions were conducted in excess $\mathrm{NaNO}_{2}$. 


$$
[\mathrm{QHOCr}(\mathrm{OH}) \mathrm{Cl}]^{+}+\mathrm{NO}_{2^{-}} \rightleftharpoons\left[\mathrm{QHOCr}(\mathrm{OH})(\mathrm{Cl}) \mathrm{NO}_{2}\right]
$$

Similar type of reactive species could be formed with thiocyanate ( $\left.\mathrm{SCN}^{-}\right)$ion to afford the $[\mathrm{QHOCr}(\mathrm{OH})(\mathrm{Cl})(\mathrm{SCN})]$ species, since the reactions are conducted in excess of $\mathrm{NH}_{4} \mathrm{SCN}$.

$$
[\mathrm{QHOCr}(\mathrm{OH}) \mathrm{Cl}]^{+}+\mathrm{SCN}^{-} \rightleftharpoons[\mathrm{QHOCr}(\mathrm{OH})(\mathrm{Cl})(\mathrm{SCN})]
$$

On the basis of foregoing discussions, mechanism of electrophilic substution in aromatic substrates could be explained through the attack of electrophile on the aromatic ring $\left(\mathrm{NO}_{2}+\right.$ released in situ from $\left[\mathrm{QHOCr}(\mathrm{OH})(\mathrm{Cl}) \mathrm{NO}_{2}\right]$ for nitration; and $\mathrm{SCN}^{+}$released in situ from [QHOCr$\left.(\mathrm{OH})(\mathrm{SCN}) \mathrm{Cl}\right]$ for thiocyanation) to afford the products, as shown in the sequence of steps of Scheme 3.

\begin{tabular}{|c|c|c|c|c|c|}
\hline \multirow[t]{2}{*}{ Entry } & \multirow[t]{2}{*}{ Product } & \multicolumn{2}{|c|}{ Conventional } & \multicolumn{2}{|c|}{ Sonication } \\
\hline & & Time (h) & Yield (\%) & Time (min) & Yield (\%) \\
\hline 1 & 4- $\mathrm{SCNC}_{6} \mathrm{H}_{4} \mathrm{NH}_{2}$ & 10 & 82 & 25 & 78 \\
\hline 2 & 2-Cl 4- $\mathrm{SCNC}_{6} \mathrm{H}_{4} \mathrm{NH}_{2}$ & 12 & 74 & 25 & 74 \\
\hline 3 & $3-\mathrm{CH}_{3} \mathrm{O} 4-\mathrm{SCNC}_{6} \mathrm{H}_{4} \mathrm{NH}_{2}$ & 11 & 77 & 20 & 77 \\
\hline 4 & 4- SCN N- $\mathrm{CH}_{3} \mathrm{C}_{6} \mathrm{H}_{5} \mathrm{NH}_{2}$ & 12 & 72 & 30 & 72 \\
\hline 5 & $\begin{array}{l}\text { 4-Thiocyanato } \mathrm{N}, \mathrm{N} \text {-dimethyl } \\
\text { aniline }\end{array}$ & 11 & 70 & 35 & 83 \\
\hline 6 & 4-Thiocyanato diphenylamine & 14 & 74 & 35 & 81 \\
\hline 7 & $2-\mathrm{SCNC}_{6} \mathrm{H}_{4} \mathrm{OH}$ & 13 & 69 & 25 & 81 \\
\hline 8 & $4-\mathrm{CH}_{3} 2-\mathrm{SCNC}_{6} \mathrm{H}_{3} \mathrm{OH}$ & 12 & 76 & 40 & 77 \\
\hline 9 & $4-\mathrm{NO}_{2} 2-\mathrm{SCNC}_{6} \mathrm{H}_{3} \mathrm{OH}$ & 14 & 80 & 40 & 72 \\
\hline 10 & $4-\mathrm{Cl} 2-\mathrm{SCNC}_{6} \mathrm{H}_{3} \mathrm{OH}$ & 14 & 77 & 30 & 87 \\
\hline 11 & $2-\mathrm{NO}_{2} 4-\mathrm{SCNC}_{6} \mathrm{H}_{3} \mathrm{OH}$ & 12 & 80 & 35 & 79 \\
\hline 12 & 2-Thiocyanato $1 \mathrm{H}$-pyrrole & 11 & 81 & 30 & 91 \\
\hline 13 & 2-Thiocyanato furan & 13 & 62 & 40 & 72 \\
\hline 14 & 2-Thiocyanato thiophene & 14 & 79 & 40 & 82 \\
\hline 15 & 3-Thiocyanato $1 \mathrm{H}$-indole & 13 & 77 & 30 & 77 \\
\hline 16 & 5-Bromo 3-thiocyanato indole & 14 & 69 & 40 & 69 \\
\hline 17 & 3-thiocyanato N-Methyl- indole & 12 & 76 & 35 & 88 \\
\hline
\end{tabular}

Table 4. Thiocyanation of aromatic compounds in presence of QDC/NH $\mathrm{NHCN}_{4}$

Table 5. Thiocyanation of aromatic compounds in presence of QCC/NH $\mathrm{NHCN}_{4}$

\begin{tabular}{clcccc} 
Entry & \multicolumn{1}{c}{ Product } & \multicolumn{2}{c}{ Conventional } & \multicolumn{2}{c}{ Sonication } \\
& & Time (h) & Yield (\%) & Time (min) & Yield (\%) \\
1 & 4- $\mathrm{SCNC}_{6} \mathrm{H}_{4} \mathrm{NH}_{2}$ & 11 & 77 & 30 & 85 \\
2 & 2-Cl 4- $\mathrm{SCNC}_{6} \mathrm{H}_{4} \mathrm{NH}_{2}$ & 10 & 84 & 35 & 83 \\
3 & 3- $\mathrm{CH}_{3} \mathrm{O} 4-\mathrm{SCNC}_{6} \mathrm{H}_{4} \mathrm{NH}_{2}$ & 10 & 78 & 45 & 81 \\
4 & 4- $\mathrm{SCN} \mathrm{N}-\mathrm{CH}_{3} \mathrm{C}_{6} \mathrm{H}_{5} \mathrm{NH}_{2}$ & 11 & 81 & 50 & 78 \\
5 & 4-Thiocyanato N, N-dimethyl & 12 & 71 & 50 & 86 \\
& aniline & & & & \\
6 & 4-Thiocyanato diphenylamine & 13 & 86 & 45 & 84
\end{tabular}




\begin{tabular}{clllll}
\hline 7 & 2-SCNC & & & \\
4 & & & \\
8 & 11 & 85 & 35 & 81 \\
8 & $4-\mathrm{CH}_{3} 2-\mathrm{SCNC}_{6} \mathrm{H}_{3} \mathrm{OH}$ & 10 & 79 & 45 & 85 \\
9 & 4- $\mathrm{NO}_{2} 2-\mathrm{SCNC}_{6} \mathrm{H}_{3} \mathrm{OH}$ & 13 & 83 & 50 & 79 \\
10 & 4-Cl2-SCNC $\mathrm{H}_{3} \mathrm{OH}$ & 13 & 77 & 35 & 82 \\
11 & 2- $\mathrm{NO}_{2} 4-\mathrm{SCNC}_{6} \mathrm{H}_{3} \mathrm{OH}$ & 11 & 82 & 45 & 89 \\
12 & 2-Thiocyanato 1H-pyrrole & 12 & 88 & 50 & 90 \\
13 & 2-Thiocyanato furan & 11 & 82 & 40 & 81 \\
14 & 2-Thiocyanato thiophene & 13 & 84 & 45 & 87 \\
15 & 3-Thiocyanato 1H-indole & 12 & 82 & 50 & 84 \\
16 & 5-Bromo 3-thiocyanato indole & 13 & 72 & 35 & 85 \\
17 & 3-thiocyanato N-Methyl-indole & 12 & 76 & 45 & 90 \\
\hline
\end{tabular}

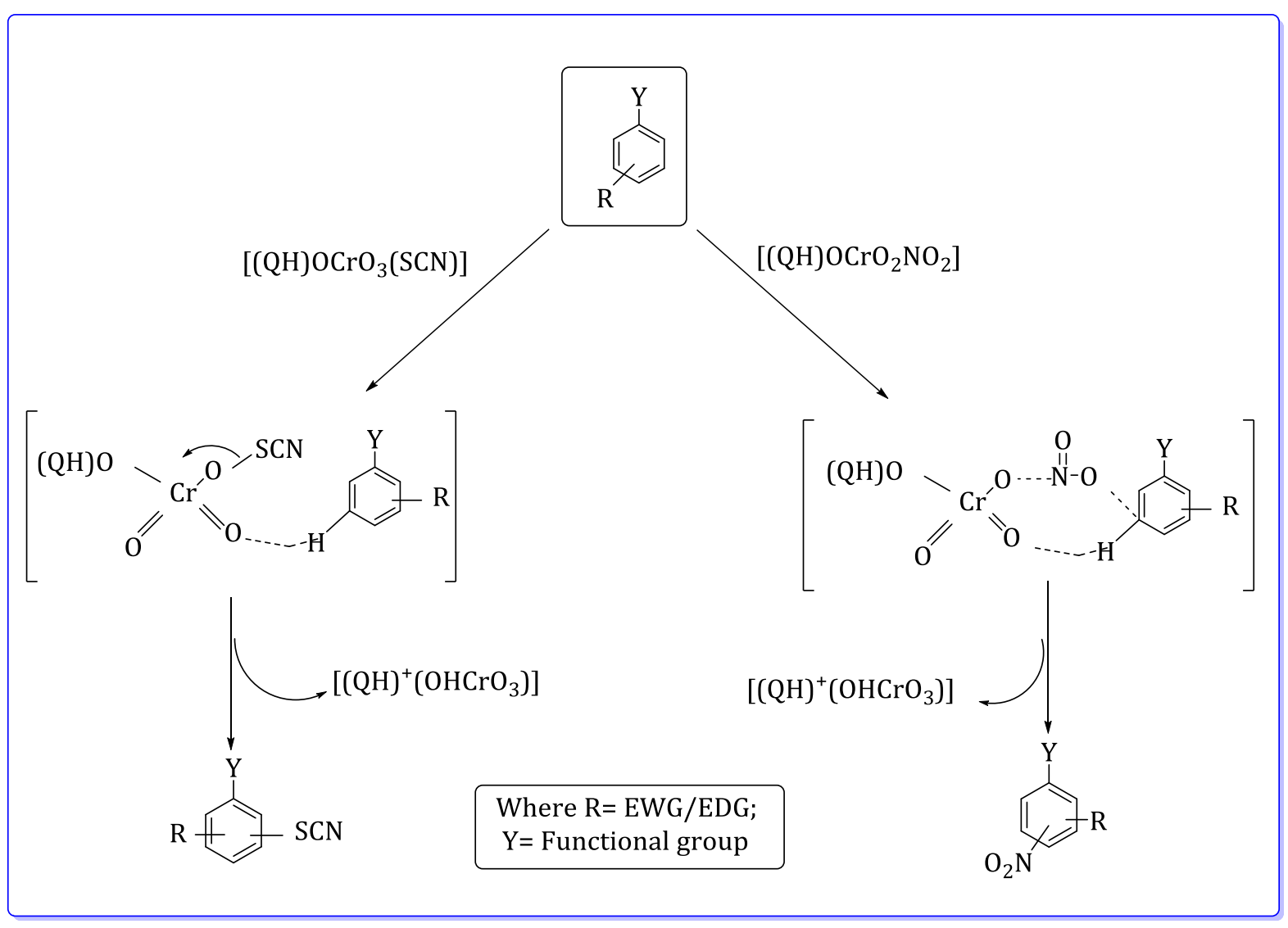

Scheme 3. QDC Electrophic aromatic substitution of aromatic compounds

\section{Salient features of ultrasonic and microwave assisted reactions}

The observed reaction times and product yields presented in Table 1, 2, 3, 4, and 5 revealed a magnificent rate enhancement in the both ultrasonically assisted nitration and thiocyanation protocols. This is basically attributed to the ultrasonic cavitation effects. The chemical effects of the ultrasound do not come from a direct interaction with the molecular species. It arises from the 
acoustic cavitation the formation, growth, and implosive collapse of bubbles in a liquid [31, 32]. Cavitation is a physical process that creates, enlarges, and implodes gaseous and vaporous cavities in an ultrasonically assisted (Irradiated) liquid. It induces very high local temperatures in the reaction mixture and enhances mass transfer [33-36]. Thus, ultrasonic assisted organic synthesis (USAOS) is a powerful green approach, which is being used to accelerate synthesis of organic compounds. It is an environmentally benign synthesis, which minimized the use of the precious metal catalysts and led to the development of new eco-friendly protocols [25]. After obtaining successful results in USA methods we were enthusiastic to see whether these reaction times could be further affected under microwave irradiation. Observed results under microwave assisted synthesis (MWAS) are compiled in Table 1 and 2 for nitration reactions. Interestingly, the reaction times further decreased enormously from several ( $\geq 25$ ) min minutes to 2-3 min), followed by high yields. Microwaves have no effect on molecular bonds or electron clouds such as infrared (IR) or the visible region of electromagnetic radiation has. This dramatic rate enhancement could be attributed to the bulk activation of the molecules, which is due to the rapid superheating of the polar solvents and pressure effects [33-36].

Quinolinium chlorochromate and quinolinium dichromate triggred nitration and thiocyanation of aromatic compounds in micellar media

Encouraged by the promising catalytic activities of micelle forming surfactants, cetyltrimethyl ammoniumbromide (CTAB), sodium dodecylsulphate (SDS), and Triton-X-100 were selected as the most promising candidates to optimize the reaction conditions for the proposed nitration and thiocyanation. The nitration of aromatic compounds in the presence of SDS underwent a rapid substitution with nitronium and thiocyanate electrophiles affording high yields of corresponding mono substituted compounds with good regioselectivity (Table 3 and 5). The observed rapid substitution with nitronium and thiocyanate electrophiles in aqueous SDS media could be due to the role of SDS micelles, which act as electrophile (Nitronium and thiocyanate ions) carriers in the nitration and thiocyanation reactions, as shown in Scheme 4. However, the reactions with CTAB and Tx-100 did not bring out any changes either in the nitration or thiocyanation reactions.

\section{Conclusion}

We have accomplished the quinolinium dichromate (QDC) and quinolinium chlorochromate (QCC) as effecient reagents for the electrophilic aromatic nitration using the sodium nitrite and thiocyanation by using ammonium thiocyanate under conventional, ultrasonic and solvent free microwave assisted conditions. The microwave assisted reactions underwent most effeciently with 


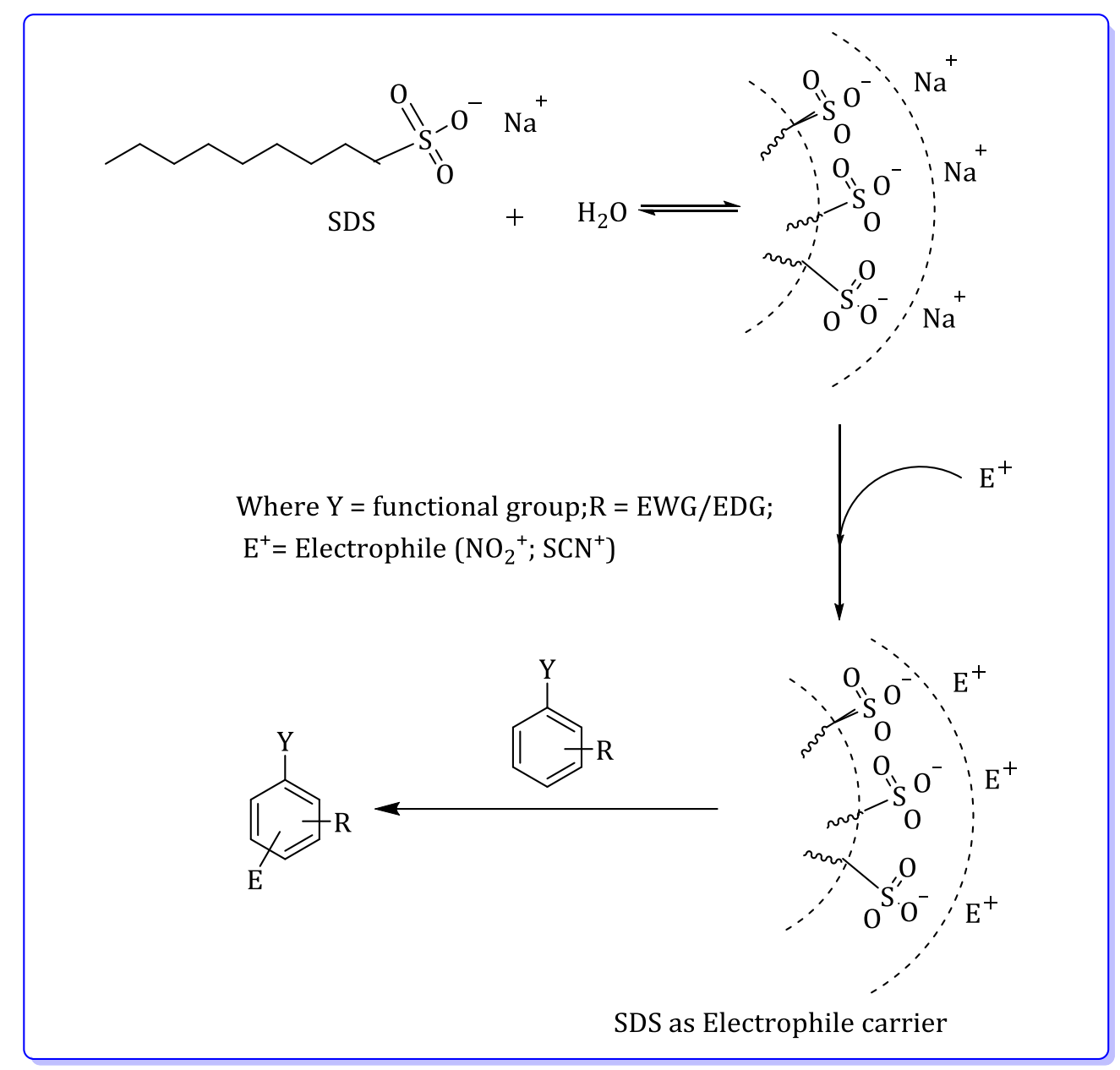

Scheme 4. SDS as electrophile carrier in electrophilic substitution of aromatic compounds very short reaction times and good product yields. Addition of anionic (SDS) micelle to the reaction medium afforded a considerable rate, while cationic (CTAB) micelle did not show much catalytic effect. These protocols have been extended to the regioselective thiocyanation of various aromatic compounds. Results were similar to the nitration protocols. However, the reactions exhibited comparatively shorter reaction times, and better yields in nitration reactions than in thiocyanation protocols.

\section{Acknowledgments}

The authors would like to appreciate the Osmania University, Hyderabad; Rayalaseema University, Kurnool, and Govt. City College, Hyderabad for their invaluable support.

\section{Disclosure statement}

No potential conflict of interest was reported by the authors. 


\section{References}

[1]. Xu X.F., Zilberg S., Haas Y.J. Phys. Chem. A, 2010, 114:4924

[2]. Olah G.A. Acc. Chem. Res., 1971, 4:240

[3]. Sana S., Rajanna K.C., Ali M.M., Saiprakash P.K. Chem. Lett., 2000, 29:48

[4]. Rodrigues J.A.R., De Oliveira A.P., Moran P.J.S., Custodio R. Tetrahedron, 1999, 55:6733

[5]. Milczak T., Jacniacki J., Zawadzki J., Malesa M., Skupinski W. Synth. Com., 2001, 31:173

[6]. Guaili W., Qiang L., Yinglin S., Wentao W., Longmin Wu. Tetrahedron Lett., 2006, 46:5831

[7]. Khazaei A., Zolfigol M.A., Mokhlesi M., Derakhshan Panah F., Sajjadifar S. Helv. Chim. Acta., 2012, 95:106

[8]. Chakrabarty M., Sarkar S. Tetrahedron Lett., 2003, 44:8131

[9]. Nair V., George T.G., Nair G., Panicker S.B. Tetrahedron Lett., 1999, 40:1195

[10]. Mahajan U.S., Akamanchi K.G. Syn. Comm., 2009, 39:2674

[11]. Akhlaghinia B., Pourali A.R., Rahmani M. Syn. Comm., 2012, 42:1184

[12]. Khalili D. Chin. Chem. Lett., 2015, 26:547

[13]. Mokhtari B., Azadi R., Rahmani-Nezhad S. Tetrahedron Lett., 2009, 50:6588

[14]. Zolfigol M.A., Khazaei A., Mokhlesi M., Vahedi H., Sajjadifar S., Pirveysian M. Phosp. Sulfur. Si. Relat. Elem., 2012, 187:295

[15]. Pan X.Q., Lei M.Y., Zou J.P., Zhang W. Tetrahedron Lett., 2009, 50:347

[16]. Nikoofar K., Gorji S. Phosp. Sulfur. Si. Relat. Elem., 2015, 190:1138

[17]. Sudhakar Chary V., Krishnaiah G., Satish Kumar M., Rajanna K.C. Phosp. Sulfur. Si. Relat. Elem., 2015, 190:1146

[18]. Venkatesham N., Rajendar Reddy K., Rajanna K.C., Veerasomaiah P. J. Sulfur Chem., 2014, 35:606 [19]. Beletskaya I.P., Sigeev A.S., Peregudov A.S., Petrovskii P.V. Mendeleev Commun., 2006, 16:250

[20]. Hemanth Sriram Y., Rajanna K.C., Satish Kumar M., Venkateswarlu M., Madhusudan Raju R. Rasayan J. Chem., 2016, 9:769

[21]. Sambashiva Rao A., Rajanna K.C., Rajendar Reddy K., Kulkarni S. Synth. React. Inorg. Metal. Org., Nano Metal Chem., 2016, 46:832

[22]. Balasubramanian K., Pratibha V. Indian J. Chem., 1986, 186, 25B:326

[23]. Narendar Reddy J., Rajanna K.C., Giridhar S., Saiprakash P.K. Trans. Metal. Chem., 1996, 21:105

[24]. Singh J., Kalsi P.S., Jawanda G.S., Chhabra B.R. Chem. Ind., 1986, 21:751

[25]. Anastas P.T., Kirchhoff, M.M. Acc. Chem. Res., 2002, 35:686

[26]. Marc Walter M., Ramaley L. Anal. Chem., 1973, 45:165

[27]. Bunton C.A. Catal. Rev., 1979, 20:1

[28]. Menger F.M. Acc. Chem. Res., 1979, 12:111 
[29]. Mallick K., Suresh J., Pradhan N., Pal T. Curr. Sci., 2001, 80:1408

[30]. La Sorella G., Strukula G., Scarso A. Green Chem., 2015, 17:644

[31]. Mason T. J. Chem. Soc. Rev., 1997, 26:443

[32]. Cella R., Stefani H.A. Tetrahedron, 2009, 65:2619

[33]. Kappe C.O. Angew Chem Int Edn., 2004, 43:6256

[34]. Dela Hoz A., Dfaz A., Moreno A. Chem. Soc. Rev., 2005, 34:164

[35]. Gedye R.N., Wei J.B. Can. J. Chem., 1998, 76:525

[36]. Varma R.S. Green Chem., 1999, 1:43

How to cite this manuscript: Satish Babu Kodali, Narendar Reddy Jakku, CHinna Rajanna Kamatala*, Rajeshwar Rao Yerraguntla, Ivon Chakravarthi Elisha. Quinolinium bound chromium(VI) reagents for efficient electrophilic aromatic nitration and thiocyanation reactions using sodium nitrate and ammonium thiocyanate. Asian Journal of Green Chemistry, 2018, 2, 70-84. DOI: 10.22631/ajgc.2017.101557.1031 